\title{
Nonlinear Analysis on Single Pile by Meshfree Local Petrov-Garlerkin Method (MLPG)
}

\author{
Ming-hua Zhao, Wei He, and You Wang \\ Geotechnial Institute, Civil engineering department, Hunan university, ChangSha, \\ 410082, China \\ Mhzhaohde21cn.com
}

\begin{abstract}
The Meshfree Local Petrov-Garlerkin Method (MLPG) was introduced to study the behavior of extra-long and large-diameter piles. The nonlinear behavior of the soil and pile shaft materials was firstly analyzed, based on these, the solving steps of MLPG was put forward involving the elastic-plastic model of soil and pile shaft reinforcement. Then a testing example was analyzed in contrast to the Finite Element Method (FEM), and the results indicated that the MLPG was of higher accuracy. Lastly, the MLPG was applied to analyze the data of static test on a full scale extra-long pile to study the behavior of pile.
\end{abstract}

Keywords: Pile foundation; Nonlinear; extra-long; MLPG method.

\section{Introduction}

For the past few years, the extra-long pile with large-diameter was more and more applied in the engineering practice. Although it takes higher bearing capacity, the fully exertion of it involved larger displacement. So it is important to study on the calculating method of settlement ${ }^{[1]}$. The finite element method (FEM) is the most highly developed tools to analyze mechanical problems, but its shape function is constructed based on the element, when analyzing the problems such as the large deflection and the contact, the serious distortion of element in FEM will cause the method unworkable. Recent years, a new numerical method termed "Meshfree Method" was put forward, in which the shape function is based on the nodes, can conquer some of the shortcomings in FEM. There are a number of meshfree methods, such as the element free Galerkin (EFG) method, the meshless local Petrov-Galerkin (MLPG) method ${ }^{[2]}$, meshless point collocation method, PCM and so on. Herein, the MLPG is studied with the method considering elastic-plastic property of the material.

\section{Basic Equation of MLPG}

In MLPG, MLS approximation is employed to create shape functions for field variable approximation, and penalty method is introduced herein to enforce the essential boundary condition. The weak form for a node, say, node $I$, based on the local weighted residual method can be stated as ${ }^{[2]}$ : 


$$
\int_{\Omega}\left(\boldsymbol{\sigma}_{i j, j}+\mathbf{b}_{i}\right) \mathbf{W}_{I} d \Omega-\alpha \int_{\Gamma_{u}}\left(\mathbf{u}_{i}-\overline{\mathbf{u}}_{i}\right) \mathbf{W}_{I} d \Gamma=0
$$

Where $\mathbf{W}_{\mathrm{I}}$ is the weight or test function, and we use the same weight function for all equations involved. The $\sigma_{\mathrm{ij}, \mathrm{j}}$ is the tensor of stress, $\mathbf{b}_{\mathrm{i}}$ is the tensor of external body force, $\mathbf{u}_{\mathrm{i}}$ is the test displacement on the boundary, $\overline{\mathbf{u}}_{i}$ is the real displacement of the node $i$ on the boundary. $\alpha$ is the penalty factor.

Using the divergence theorem, we obtain:

$$
\int_{\Gamma_{s}} \sigma_{i j} n_{j} \mathbf{W}_{I} \mathrm{~d} \Gamma_{s}-\int_{\Omega_{s}} \sigma_{i j} \mathbf{W}_{I, j} \mathrm{~d} \Omega_{s}+\int_{\Omega_{s}} \mathbf{b}_{i} \mathbf{W}_{I} \mathrm{~d} \Omega_{s}-\alpha \int_{\Gamma_{u s}}\left(\mathbf{u}_{i}-\overline{\mathbf{u}}_{i}\right) \mathbf{W}_{I} \mathrm{~d} \Gamma_{u s}=0
$$

Where $\Gamma_{s}=L_{s} \cup \Gamma_{u s} \cup \Gamma_{u t}$.

Then the formula (2) can be transformed to:

$$
\begin{aligned}
& \int_{\Omega_{s}} \boldsymbol{\sigma}_{i j} \mathbf{W}_{I, j} \mathrm{~d} \Omega_{s}+\alpha \int_{\Gamma_{u s}} \mathbf{u}_{i} \mathbf{W}_{I} \mathrm{~d} \Gamma_{u s}-\int_{\Gamma_{s u}} \boldsymbol{\sigma}_{i j} \mathbf{n}_{j} \mathbf{W}_{I} \mathrm{~d} \Gamma_{s u}-\int_{L_{s}} \boldsymbol{\sigma}_{i j} \mathbf{n}_{j} \mathbf{W}_{I} \mathrm{dL} L_{s} \\
& =\int_{\Gamma_{s t}} \boldsymbol{\sigma}_{i j} \mathbf{n}_{j} \mathbf{W}_{I} \mathrm{~d} \Gamma_{s t}+\int_{\Omega_{s}} \mathbf{b}_{i} \mathbf{W}_{I} \mathrm{~d} \Omega_{s}+\alpha \int_{\Gamma_{u s}} \overline{\mathbf{u}}_{i} \mathbf{W}_{I} \mathrm{~d} \Gamma_{u s}
\end{aligned}
$$

When the calculating domain is located entirely in the problem domain, integrals related to $\Gamma_{u s}$ and $\Gamma_{u t}$ vanish, and the formula (3) can be simplified as:

$$
\int_{\Omega_{s}} \boldsymbol{\sigma}_{i j} \mathbf{W}_{I, j} \mathrm{~d} \Omega_{s}=\int_{\Omega_{s}} \mathbf{b}_{i} \mathbf{W}_{I} \mathrm{~d} \Omega_{s}
$$

\section{Elastic-Plastic Models of Soil and Reinforcement Concrete}

\subsection{Elastic-Plastic Model for Soil}

The yield rule can be described as the function of stress in the soil. That is:

$$
f^{*}\left(\sigma_{i j}\right)=k_{f}
$$

Where $f^{*}\left(\sigma_{\mathrm{ij}}\right)$ is the function of stress, can use a certain form of stress invariant as independent variable.

The rule put forward by Drucker and Prager is a form of the general Mises rule ${ }^{[3]}$ :

$$
\alpha I_{1}+\sqrt{J_{2}}=k
$$

Where $J_{2}$ is the second stress invariant, it is

$$
J_{2}=\frac{1}{6}\left[\left(\sigma_{1}-\sigma_{2}\right)^{2}+\left(\sigma_{2}-\sigma_{3}\right)^{2}+\left(\sigma_{3}-\sigma_{1}\right)^{2}\right]
$$

Drucker and Prager also got the parameter in planar stress conditions:

$$
\alpha=\frac{\sin \varphi}{\sqrt{3} \sqrt{3+\sin ^{2} \varphi}} \quad k=\frac{\sqrt{3} c \cos \varphi}{\sqrt{3+\sin ^{2} \varphi}}
$$

Where the $c$ and $\varphi$ is the cohesion and inner friction angle. When $\varphi=0$, the formula (6) turns to be Mises rule. 


\subsection{Elastic-Plastic Model for Pile Shaft Reinforcement Concrete}

The behavior of reinforcement concrete is quite different from the soil, and the Japanese scholar Noguchi ${ }^{[4]}$ advised that the $\alpha$ and $k$ can be got as:

$$
\alpha=0.07, k=-\frac{0.88}{\sqrt{3}}\left|f_{c}^{\prime}\right|
$$

Where the $f_{\mathrm{c}}{ }^{\prime}$ is the compression strength of prism shape concrete.

\section{Testing Example}

The cantilever beam acted by a vertical fixate was analyzed by MLPG, FEM and elastic solution ${ }^{[5]}$. The beam is pictured in Fig. 1, and the result is shown in Table 1.

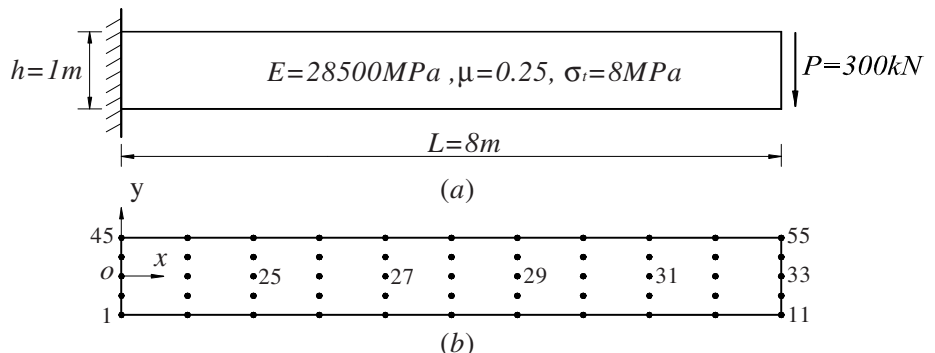

Fig. 1. Calculating model of vertical loaded beam

Table 1. The displacement of y direction at each node by three method

\begin{tabular}{cccccccc}
\hline No. & $\begin{array}{c}\text { Elastic } \\
\text { Solution }\end{array}$ & Marc & Difference & Precise & MLPG & Difference & Precise \\
\hline 23 & 0.000 & 0.000 & - & - & 0 & - & - \\
24 & 0.296 & 0.251 & -0.044 & -0.15 & 0.321 & 0.026 & 0.09 \\
25 & 1.205 & 0.975 & -0.230 & -0.19 & 1.257 & 0.052 & 0.04 \\
26 & 2.632 & 2.107 & -0.525 & -0.20 & 2.727 & 0.095 & 0.04 \\
27 & 4.511 & 3.599 & -0.913 & -0.20 & 4.673 & 0.161 & 0.04 \\
28 & 6.779 & 5.398 & -1.381 & -0.20 & 7.003 & 0.224 & 0.03 \\
29 & 9.370 & 7.454 & -1.915 & -0.20 & 9.697 & 0.327 & 0.03 \\
30 & 12.219 & 9.716 & -2.504 & -0.20 & 12.658 & 0.439 & 0.04 \\
31 & 15.263 & 12.131 & -3.132 & -0.21 & 15.816 & 0.553 & 0.04 \\
32 & 18.436 & 14.649 & -3.787 & -0.21 & 19.108 & 0.672 & 0.04 \\
33 & 21.674 & 17.221 & -4.453 & -0.21 & 22.469 & 0.795 & 0.04 \\
\hline
\end{tabular}

\section{Analysis on a Test Pile by MLPG Method}

A test pile ${ }^{[6]}$, which was 60 meters long, and the calculating domain could be determined as a rectangle domain which was 120 meters high and 120 meters long. 
Because of the large slenderness ratio, the domain had to be represented by huge number of scattered nodes, 26225 nodes were used here. The contour map of the calculating domain can be got by MLPG, and due to the limit of the space, only principal strain $\mathcal{E}_{1}$ is shown in Fig.2

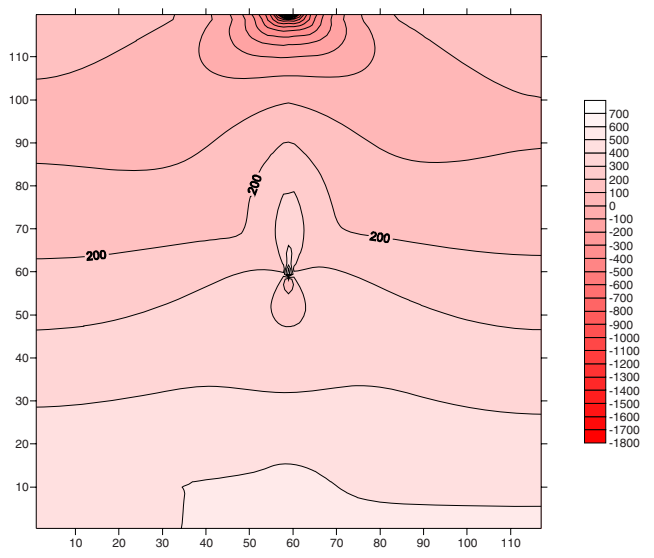

Fig. 2. Contour map of principal strain $\mathcal{E}_{1}$

\section{Conclusions}

The conclusion can be drawn as follows:

1. The basic equation of MLPG was deduced by weighted residual method, and the nonlinear behavior of the soil and pile were studied.

2. The cantilever beam, was analyzed by MLPG, FEM and elastic solution. The results of these three methods showed that the MLPG had better accuracy than FEM.

3. A test pile was analyzed by MLPG, and contour maps of strain in the problem domain could be got.

\section{References}

1. Zhao Minghua. Calculating and Detecting for Pile Foundations of Bridges $[\mathrm{M}]$. Beijing: China Communications Press, 2000. (in Chinese)

2. Atluri SN, Zhu T. A new meshless local Petrov-Galerkin (MLPG) approach in computational mechanics. Comput. Mech., 1998, 22:117-127

3. Qian Jiahuan, Yin Zongze. Principle and calculation of soil (The $2^{\text {nd }}$ edition) $[\mathrm{M}]$. Beijing: China water conservancy and electrical power press, 1995 (in Chinese)

4. Lv Xilin, Jin Guofang, Wu Xiaohan. Nonlinear theory and application of FEM for reinforcement concrete. Shanghai: Tongji university press, 1997 (in Chinese)

5. Xu Zhilun. Abecedarium for elastic mechanics. Beijing: High education press. 1983 (in Chinese)

6. Zhao Ming-hua, Zou Xin-jun, Liu Qi-jian. Research on the axial bearing capacity of superlong and large diameter bored piles in Dongting Lakeland by loading test [J]. China civil engineering journal. 2004,37(10): 63-67 (in Chinese) 